\title{
Ideação suicida em universitários da área da saúde: prevalência e fatores associados
}

\author{
Suicidal ideation among health field undergraduates: prevalence and associated factors \\ Ideación suicida en universitarios del área de la salud: prevalencia y factores asociados
}

\section{Como citar este artigo:}

Veloso LUP, Lima CLS, Sales JCS, Monteiro CFS, Gonçalves AMS, Silva Júnior FJG. Ideação suicida em universitários da área da saúde: prevalência e fatores associados. Rev Gaúcha Enferm. 2019;40:e20180144. doi: https://doi.org/10.1590/19831447.2019.20180144.

\footnotetext{
a Universidade Federal do Piauí (UFPI), Programa de Pós-Graduação em Enfermagem. Teresina, Piauí, Brasil.

b Universidade Estadual do Piauí (UESPI). Teresina, Piauí, Brasil.

' Universidade Federal do Piauí (UFPI), Mestrado Profissional em Saúde da Família, Departamento de Enfermagem. Teresina, Piauí, Brasil.

'Universidade Federal de São Carlos (UFSCAR) Programa de Pós-Graduação em Enfermagem. São Carlos, São Paulo, Brasil.
}

\section{RESUMO}

Objetivo: Identificar prevalência e fatores associados da ideação suicida em universitários da área da saúde.

Método: Estudo transversal, com 142 universitários da área da saúde de instituição pública de Teresina-Piauí-Brasil. Utilizou-se Escala de Ideação Suicida e questionário para caracterização. Realizou-se teste qui-quadrado, exato de Fisher, cálculo da Odds-Ratio, Kruskal-Wallis e correlação de Spearman. Adotou-se nível de significância de 0,05.

Resultados: A prevalência de ideação suicida foi 22\%, sobretudo, entre homens, solteiros e com vínculo empregatício. Uso de álcool, tabaco e outras drogas, histórico de bullying, tentativa de suicídio e não estar no curso desejado estão associados a ideação suicida. Observou-se que quanto maior o escore da escala menor o rendimento acadêmico. Universitários do curso de psicologia possuem maior extensão da motivação e planejamento do comportamento suicida.

Conclusão: Alta prevalência de ideação suicida entre universitários da área de saúde e fatores associados reforçam a necessidade de estratégias de intervenção nas universidades.

Palavras chaves: Ideação suicida. Estudantes de ciências da saúde. Saúde Mental.

\section{ABSTRACT}

Objective: To identify the prevalence of suicidal ideation and its associated factors in university students from the health field, in a public higher education institution.

Method: Cross-sectional study with 142 university students. Suicide Ideation Scale and a questionnaire for characterization were used. Fischer's exact test, chi-square, Odds-Ratio, Kruskal-Wallis, and Spearman's correlation were performed. A significance level of 0.05 was adopted.

Results: The prevalence of suicidal ideation was $22 \%$. It was associated to the use of alcohol, tobacco, and other drugs, bullying, suicide attempts ( $p$-value $=0.000)$, and not attending the course desired ( $p$-value $=0.001$ ). It was observed that the higher the score the lower the academic performance ( $p$-value $=0.000$ ). There was a difference in the mean score according to course ( $p$-value $=0.000)$, and the score was higher among psychology students.

Conclusion: The high prevalence of suicidal ideation among university students in the health field reiterates the need to create intervention strategies, especially in the context of universities.

Keywords: Suicidal ideation. Students, health occupations. Mental health.

\section{RESUMEN}

Objetivo: Identificar prevalencia de la ideación suicida en universitarios, del área de la salud, de institución de enseñanza superior pública y factores asociados.

Método: Estudio transversal, con 142 universitarios. Se utilizó Escala de Ideación Suicida y cuestionario para caracterización. Se realizó una prueba Chi-cuadrado, exacta de Fisher, cálculo de la Odds-Ratio, Kruskal-Wallis y correlación de Spearman. Se adoptó un nivel de significancia de 0,05.

Resultados: La prevalencia de ideación suicida fue $22 \%$ y estuvo asociada al uso de alcohol, tabaco y otras drogas, ser víctima de bullying, tener historial de intento de suicidio ( $p$-valor $=0,000)$ y no frecuentar el curso que desea $(p=0,001)$. Se observó que cuanto mayor sea la puntuación de la escala menor rendimiento académico $(p$-valor $=0,000)$. Hay diferencia en el promedio de la puntuación por curso ( $p$-valor $=0,000$ ), siendo mayores en el curso de psicología.

Conclusión: La alta prevalencia de ideación suicida entre universitarios del área de salud refuerza la necesidad de crear estrategias de intervención, principalmente, en el contexto de las universidades.

Palabras clave: Idea suicida. Estudiantes del área de la salud. Salud Mental. 


\section{—INTRODUÇÃO}

O ingresso no ensino superior representa fase de mudanças no convívio social e nas atividades cotidianas, proporciona autonomia e liberdade ao universitário, mas também implica em responsabilidades frente às cobranças acadêmicas, independente de gênero, idade ou meio cultural. Aqueles cujo processo de formação é na área da saúde vivenciam cotidianamente dilemas específicos da profissão escolhida que envolve a dualidade entre saúde/ doença e vida/morte ${ }^{(1)}$.

Esta realidade pode afetar seu desempenho acadêmico e ter como consequência resultados negativos, em que se evidencia o estado de desequilíbrio e o sentimento de inabilidade para resolução dos conflitos, o que pode desencadear problemas de saúde mental, tendo como consequência o comportamento suicida ${ }^{(2)}$.

Esse comportamento refere-se à conduta da pessoa que busca se ferir ou se matar. A ideação suicida faz parte do espectro do comportamento suicida, que inclui ainda o planejamento para suicídio, tentativa de suicídio e suicídio em si(3).

Dados da Organização Mundial de Saúde (OMS) demonstram que mais de 800 mil suicídios ocorrem anualmente em todo o mundo. Essa problemática é considerada a segunda principal causa de morte entre jovens com idade entre 15 e 29 anos $^{(4)}$ - faixa etária em que a maioria dos universitários estão inseridos. Neste contexto, chama atenção para ideação suicida, principalmente, entre jovens ${ }^{(5)}$, pois sua progressão, avaliada por meio de informações acerca do planejamento claro e intencionalidade da pessoa, podem sinalizar efetivação do suicídio nas próximas horas ou dias( ${ }^{(6)}$.

A ideação suicida em universitários configura-se em campo pouco explorado e marginalizado, o que demonstra a necessidade de desenvolvimento de novos conhecimentos $^{(7)}$. No Brasil, especificamente, estudos sobre essa temática encontram-se centralizados na região Sul, Sudeste e Centro-Oeste, locais em que são registrados maiores taxas de suicídios ${ }^{(8)}$. Além disso, reconhecer a prevalência da ideação suicida e fatores associados representa estratégia efetiva de prevenção de desfecho trágico qual seja o suicídio.

Diante deste contexto, tem-se como questão norteadora: qual a prevalência e fatores associados à ideação suicida em universitários, da área da saúde, de instituição de ensino superior pública? Para tanto, definiu-se como objetivo deste estudo identificar prevalência e fatores associados da ideação suicida em universitários da área da saúde.

\section{口 MÉTODO}

Estudo transversal realizado em instituição pública de ensino superior do Estado do Piauí-Brasil, que contempla quatro cursos, na área da saúde: enfermagem, fisioterapia, medicina e psicologia.

A população constou de 849 universitários. Para o cálculo da amostra foi utilizado nível de confiança de 95\%, erro máximo de $5 \%$ e prevalência presumida de ideação suicida de $12,6 \%{ }^{(9)}$, ao que se obteve amostra de 142 universitários. Utilizou-se técnica de amostragem probabilística aleatória simples considerando que a quantidade de universitários por curso era similar.

Foram critérios de inclusão: universitários, de ambos os sexos, regularmente matriculados nos referidos cursos e com idade igual ou superior a 18 anos. Como critérios de exclusão: universitários que estivessem afastados das atividades acadêmicas por motivo de doença ou que estavam em intercâmbios institucionais. Destaca-se que não houveram perdas da amostra calculada.

A coleta de dados ocorreu no período de janeiro a abril de 2017. Realizou-se recrutamento dos universitários por meio de sorteio a partir do software Excel 2010, considerando o número de matrícula disponível no sistema acadêmico.

Foi aplicado questionário estruturado que possibilitou caracterização da amostra quanto às variáveis sociodemográficas, econômicas, acadêmicas e acerca de eventos da vida como uso de álcool, tabaco e outras drogas, bullying, histórico de tentativa de suicídio.

Os dados sobre ideação suicida foram obtidos a partir da Escala de Ideação Suicida de Beck (BSI), composta por 21 itens, sendo que os primeiros 19 apresentam três alternativas de respostas que indicam progressão do agravamento de desejos, atitudes e planos suicidas. O escore total pode variar de 0 a 38, sendo calculado a partir do somatório dos 19 primeiros itens. O fato de ocorrer à presença de pontuação no BSI, já qualifica a pessoa como vítima do comportamento suicida, em especial a ideação. Destaca-se que a BSI é validada no Brasil com a população geral (não clínica) de adolescentes ${ }^{(10)}$.

Para testar o questionário e avaliar habilidades dos pesquisadores no manuseio da referida escala, foi realizado teste piloto com 10\% da amostra. As informações oriundas desta etapa não compuseram o banco de dados para análise.

Os instrumentos de coleta de dados foram entregues em envelopes aos universitários e recebidos após o preenchimento. Os participantes foram esclarecidos quanto ao objetivo, método do estudo e convidados a assinar o Termo de Consentimento Livre e Esclarecido (TCLE). Essa estratégia garantiu confidencialidade e privacidade conforme as diretrizes éticas e legais das Resoluções no. 466/2012 e no. 510/2016. O estudo recebeu aprovação do Comitê de Ética de Pesquisa da Universidade Estadual do Piauí (Parecer n. 1.861.528), em 07 de dezembro de 2016. 
Os dados foram submetidos à análise no software SPSS, versão 20.0. Realizou-se estatística descritiva (frequência absoluta, porcentagem, média e desvio padrão). Para verificar associação entre variáveis qualitativas foi utilizado teste qui-quadrado $\left(c^{2}\right)$ e exato de Fisher. A força das associações entre as variáveis foi aferida pela Odds-Ratio (OR) e intervalos de confiança (IC 95\%). O teste de Kolmogorov-Smirnov foi aplicado nas variáveis numéricas que possibilitou verificar distribuição não paramétrica dos dados. Para comparação de médias do rendimento acadêmico entre os universitários dos quatro cursos foi utilizado teste Kruskal-Wallis e para estudo das associações entre o escore do BSI e rendimento acadêmico, utilizou-se o coeficiente de correlação de Spearman. Adotou-se nível de significância de 0,05 para todos os testes realizados.

\section{RESULTADOS}

Observou-se que 22\% dos universitários apresentaram ideação suicida, sendo a média do BSI de 4,08 $( \pm=8,10)$.
Dentre aqueles que apresentaram ideação suicida, 58,1\% eram do sexo masculino, $71,0 \%$ solteiros, 58,1\% procedentes de Teresina, 74,2\% moravam acompanhados (familiares ou amigos), 90,3\% possuíam renda familiar maior que um salário mínimo e 67,8\% vínculo empregatício. Na análise bivariada, essas variáveis não apresentaram associação estatisticamente significativa com a presença de ideação suicida ( $p$-valor>0,05).

Entre os universitários com presença de ideação suicida 90,3\% relataram uso de bebida alcoólica, 54,8\% de tabaco e $54,8 \%$ de outras drogas. Essas variáveis apresentaram associação significativa com a presença de ideação suicida. O uso de álcool, tabaco e outras drogas aumentam em 7,11 (IC=2,04-24,78), 9,15 (IC=3,67-22,82) e 11,03 (IC=4,3028,32), respectivamente, as chances dos universitários apresentarem ideação suicida. Ter sido vítima de bullying aumenta em 9,55 vezes (IC=3,904-23,389) e possuir histórico de tentativa de suicídio em 243 vezes as chances de ideação suicida (IC=51,308-1150,874) (Tabela 1).

Tabela 1 - Associação entre ideação suicida com uso de álcool, tabaco e outras drogas, vítima de bullying e histórico de tentativas de suicido entre universitários da área da saúde. Teresina, 2017. (n=142)

\begin{tabular}{|c|c|c|c|c|c|c|c|}
\hline & Pre: & ença de id & ação & uicida & & & \\
\hline & & Sim & & ào & OR & IC & p-valor \\
\hline & $\mathbf{N}$ & $\%$ & $\mathbf{N}$ & $\%$ & & & \\
\hline Uso de álcool & & & & & 7,11 & $2,04-24,78$ & $0,000^{* *}$ \\
\hline Sim & 28 & 90,3 & 63 & 56,8 & & & \\
\hline Não & 3 & 9,7 & 48 & 43,2 & & & \\
\hline Uso de tabaco & & & & & 9,15 & $3,67-22,82$ & $0,000^{*}$ \\
\hline Sim & 17 & 54,8 & 13 & 11,7 & & & \\
\hline Não & 14 & 45,2 & 98 & 88,3 & & & \\
\hline Uso de outra droga & & & & & 11,03 & $4,30-28,32$ & $0,000^{*}$ \\
\hline Sim & 17 & 54,8 & 11 & 9,9 & & & \\
\hline Não & 14 & 45,2 & 100 & 90,1 & & & \\
\hline Vítima de bullying & & & & & 9,55 & $3,904-23,389$ & $0,000^{*}$ \\
\hline Sim & 21 & $67,832,2$ & 20 & 18,0 & & & \\
\hline Não & 10 & & 91 & 82,0 & & & \\
\hline $\begin{array}{l}\text { Histórico de tentativa } \\
\text { de suicídio }\end{array}$ & & & & & 243,00 & $51,308-1150,874$ & $0,000^{* *}$ \\
\hline Sim & 27 & 87,1 & 3 & 2,7 & & & \\
\hline Não & 4 & 12,9 & 108 & 97,3 & & & \\
\hline
\end{tabular}

Fonte: Dados da pesquisa, 2017

Legenda: *Teste qui-quadrado; ** Teste Exato de Fischer 
Observou-se que existe associação estatisticamente significativa entre ideação suicida e curso desejado ( $p$ -valor $=0,001$ ), sendo que $80,6 \%$ dos universitários que tem ideação suicida não frequentam o curso que deseja. Não frequentar o curso que deseja aumenta em 4,72 vezes as chances de ter ideação suicida (IC=1,8-12,4).

Há correlação negativa entre o escore do BSI com o rendimento acadêmico ( $p$-valor $=0,000$ ), ou seja, quanto maior o escore BSI menor o rendimento acadêmico.

Observou-se, também, que há diferença estatisticamente significativa na média do escore do BSI por curso, sendo o curso de psicologia aquele que apresentou maior média no escore do BSI (Média=11,48; Desvio Padrão=10,55).

\section{DISCUSSÃO}

A prevalência de pensamentos suicidas entre os universitários pesquisados é considerada alta. Neste estudo, os testes de associação permitiram achados significativos, principalmente quanto as variáveis uso de bebida alcoólica, do tabaco e de outras drogas, ter histórico de bullying e já ter tentado suicídio, bem como não fazer o curso desejado. Esses fatores aumentam as chances de ter ideação suicida, demonstrando que essas variáveis se associam de modo consistente com a ideação suicida.

Outro achado relevante foi que quanto mais intensa é a ideação suicida, menor o rendimento acadêmico. Chama atenção também que entre os cursos acadêmicos, o de psicologia apresentou maior média, nos quais os alunos apresentam ideação suicida, seguido pelo curso de medicina.

Esses dados trazem preocupantes reflexões que vão desde a escolha do curso, a entrada na universidade, as relações que ali se estabelecem e as exigências do próprio ambiente acadêmico.

O acesso à universidade representa momento de desafios para universitários, que envolve o desempenho pessoal, acadêmico e social, além de demandar maturidade, tempo, dedicação e exercício da autonomia. Entretanto, quando há dificuldades relacionadas a essas demandas pode-se observar sentimentos de preocupação, incerteza, tristeza, fracasso e não familiaridade com a própria vida, que podem progredir para pensamentos depressivos e incluir ideias em tirar a própria vida(11).

Alguns estudos sugerem que a provável existência de diferentes agentes estressores, ao longo dos cursos da área da saúde, tais como: perda da liberdade pessoal, alto nível de exigência e de conteúdo, sentimento de desumanização, falta de tempo para lazer, forte competição entre colegas e o próprio contato com pacientes, podem contribuir também para o surgimento de sintomas depressivos que, por sua vez, repercutirão no desempenho acadêmico e nos relacionamentos sociais ${ }^{(12-13)}$.

Acrescenta-se que o ambiente universitário possibilita novas descobertas e vínculos afetivos e relacionais, bem como participação em círculos sociais por meio de festas. Entretanto, para ser aceito neste meio, é comum que jovens universitários adotem posturas vulneráveis dentre elas o uso de álcool, tabaco e outras drogas. Os achados desse estudo mostram associação entre o uso dessas substâncias e a presença de pensamentos suicidas.

Há evidências de especificidades quanto ao uso de drogas lícitas e sua relação com o comportamento suicida, de modo que enquanto o consumo de álcool é frequentemente associado a ideação, o consumo abusivo de tabaco está relacionado as tentativas de suicídio(14).

No presente estudo, os achados mostraram relação da ideação suicida com bullying. Nesse sentido, alguns estudos apontam que o bullying tem efeito indireto sobre o comportamento suicida, pois pode deflagrar sintomas depressivos e favorecer o uso de álcool, tabaco e outras drogas, resultando em sofrimento psicológico, prejuízo social, profissional e outras dimensões importantes da vida. Essas situações traumáticas impactam negativamente na saúde mental do indivíduo e aumentam a probabilidade do comportamento suicida em seis vezes quando comparado com a população gera|(15-16).

Outro achado deste estudo foi o histórico de tentativa de suicídio. Sobre a associação dessa variável com ideação suicida, pesquisa mostra que história de tentativas de suicídio possui valor preditivo na avaliação do risco de suicídio, uma vez que a reincidência do comportamento suicida normalmente é observada por meio de pensamentos recorrentes de morte ${ }^{(1)}$.

Nesta perspectiva, a identificação e o acompanhamento de universitários com histórico de tentativa de suicídio deve ser priorizado no espaço da universidade, de modo que direcione atenção e condutas de professores, servidores e demais alunos, com vistas ao fortalecimento de vínculos e da escuta qualificada.

Demonstra-se, nesse estudo, que o aluno não cursar a graduação desejada associa-se à ideação suicida. Muitas vezes estudantes que não lograram êxito no acesso a universidade para o curso desejado, mas iniciam outra carreira pela necessidade de atender expectativas familiares em detrimento das suas e ou por imposições sociais relacionadas ao que se consideram carreiras ascendentes, que promovem maior status e rápido retorno financeiro, levam a sentimentos de frustração e incapacidade.

Além disso, deve-se considerar que o modo como o universitário percebe o mundo e lida com os fatores 
estressores na academia, como sentimentos de não aceitação no curso escolhido, carga horária exaustiva e insegurança relacionada ao futuro profissional, repercutem na saúde mental que inclui entre as consequências mais graves a ideação suicida ${ }^{(9,17)}$.

Por sua vez, este estudo aponta que pensamentos em tirar a própria vida refletem negativamente no rendimento acadêmico dos universitários. Estudantes com estes pensamentos, comumente, deflagrados por problemas de saúde mental apresentam falhas relacionadas às suas obrigações acadêmicas que incluem: atrasos e ou ausências e ou abandono, bem como dificuldade de concentração, que refletem no processo de ensino-aprendizagem e, consequentemente, no rendimento acadêmico.

Alguns estudos afirmam que são observados déficits no rendimento acadêmico de universitários com problemas de natureza psiquiátrica, a exemplo da Depressão Maior $(10,3 \%)$ e comportamento suicida $(10,3 \%)^{(18-19)}$. Portanto, esses dados apresentados sugerem a existência de relações complexas entre estas variáveis e reforçam a necessidade de se articular adequadamente os diferentes domínios da vida da pessoa quando se desenham estratégias de intervenção.

Chama atenção nos dados levantados nesse estudo que existem diferenças na ocorrência de pensamentos em tirar a própria vida quando analisados os universitários dos quatro cursos descritos, sendo que o escore do BSI foi mais elevado entre os estudantes do curso de psicologia.

Os dados referentes ao curso de psicologia apresentam semelhança a estudo realizado na universidade da Colômbia, com 1265 estudantes de psicologia em que 3,6\% apresentaram alto risco de suicídio e 9,1\% automutilação pelo menos uma vez na vida e que para cada aluno que apresentou um risco mínimo de automutilação seis alunos se feriram de maneira séria, o que reforça a ideia em tirar a própria vida(20).

De modo geral, os universitários da área da saúde vivenciam no decorrer da graduação estímulos emocionais intensos, principamente, relacionados à dualidade vida/ morte comumente observados no processo de cuidar de pessoas, além das inúmeras exigências que, embora, não obrigatórias, atualmente, são consideradas importantes no processo de formação profissional. Essas situações tendem a contribuir para o aparecimento de dificuldades interpessoais e surgimento de sintomas de angústia, tristeza, incapacidade, ansiedade, sentimento de não pertencimento que, por sua vez, podem deflagrar ideias suicidas.

\section{口CONCLUSÃO}

A prevalência de ideação suicida entre universitários foi de $22 \%$ e os fatores associados foram: uso de álcool, tabaco e outras drogas, ser vítima de bullying, ter histórico de tentativa de suicídio e não frequentar o curso que deseja.

Os universitários com maior escore do BSI tiveram menor rendimento acadêmico. Além disso, há diferença na média dos escores, por curso, sendo que os maiores escores do BSI foram referentes aos universitários do curso de psicologia.

O presente estudo apresenta como limitações ter sido realizado com amostra não clínica, ter utilizado medidas de autorrelato e o próprio delineamento do estudo, que impossibilita avaliar relação causal.

Considerando os fatores associados a ideação suicida entre universitários espera-se que este estudo possa contribuir para criação de sistemas integrados de cuidados de saúde, no contexto das universidades, contemplando ações de apoio físico, mental e de saúde acadêmica, a exemplo da escuta qualificada por enfermeiros e docentes, com vistas a oferecer soluções para os universitários.

\section{口EFERÊNCIAS}

1. Gómez-Romero MJ, Limonero JT, Trallero JT, Montes-Hidalgo J, Tomás-Sábado J. Relationship between emotional intelligence and negative affect on suicide risk in young university students. Ansiedad Estrés. 2018;24(1):18-23. doi: https:// doi.org/10.1016/j.anyes.2017.10.007.

2. Stoliker B, Lafreniere K. The influence of perceived stress, Ioneliness, and learning burnout on university students' educational experience. Coll Stud J. 2015 [cited 2018 Jan 22];49(1):146-60. Available from: https://www.researchgate.net/ publication/282817514_The_Influence_of_Perceived_Stress_Loneliness_ and_Learning_Burnout_on_University_Students'_Educational_Experience

3. Souza MMC, Bastos FAE, Mangas RMN. 0 comportamento suicida de idosos institucionalizados: histórias de vida. Physis. 2017;27(4):981-1002. doi: https:// doi.org/10.1590/s0103-73312017000400007.

4. World Health Organization (CH) [Internet]. Geneva: WH0, c2018-2019 [cited 2019 Apr 25]. Suicide; [about 1 screen]. Available from: https://www.who.int/ en/news-room/fact-sheets/detail/suicide.

5. Ribeiro JM, Moreira MR. An approach to suicide among adolescents and youth in Brazil. Ciênc Saúde Coletiva. 2018;23(9):2821-34. doi: https://doi. org/10.1590/1413-81232018239.17192018.

6. Del-Bem CM, Sponholz Junior A, Montovani C, Faleiros MCM, Oliveira GEC, Guapo VG. Emergências psiquiátricas: manejo de agitação psicomotora e avaliação de risco suicida. Medicina (Ribeirão Preto). 2017;50(1):98-112. doi: https://doi.org/10.11606/issn.2176-7262.v50isupl1.p98-112.

7. Cano-Montalbán I, Quevedo-Blasco R. Sociodemographic variables most associated with suicidal behaviour and suicide methods in Europe and America. a systematic review. Eur J Psychol Applied Legal Context. 2018;10(1):15-25. doi: https://doi.org/10.5093/ejpalc2018a2.

8. Calixto Filho M, Zerbini T. Epidemiologia do suicídio no Brasil entre os anos de 2000 e 2010. Saúde Ética Justiça. 2016 [citado 2018 fev 07];21(2):45-51. 
Disponível em: https://www.revistas.usp.br/sej/article/view/134006.

9. Pereira A, Cardoso F. Suicidal ideation in university students: prevalence and association with school and gender. Paidéia (Ribeirão Pret0). 2015;25(62):299306. doi: https://doi.org/10.1590/1982-43272562201503.

10. Cunha JA. Manual da versão em português das Escalas de Beck. São Paulo: Casa do Psicólogo; 2001.

11. Nyer M, Holt DJ, Pedrelli P, Fava M, Ameral V, Cassiello CF, et al. Factors that distinguish college students with depressive symptoms with and without suicidal thoughts. Ann Clin Psychiatry. 2013 [cited 2018 May 12];25(1):41-9. Available from: https://www.ncbi.nlm.nih.gov/pmc/articles/PMC3791316/.

12. Cybulski C, Mansani FP. Análise da depressão, dos fatores de risco para sintomas depressivos e do uso de antidepressivos entre acadêmicos do curso de medicina da Universidade Estadual de Ponta Grossa. Rev Bras Educ Med. 201741(1):92101. doi: https://doi.org/10.1590/1981-52712015v41n1rb20160034.

13. Auerbach RP, Alonso J, Axinn WG, Cuijpers P, Ebert DD, Green JG, et al. Mental disorders among college students in the World Health Organization World Mental Health Surveys. Psychol Med. 2016;46(14):2955-70. doi: https://doi. org/10.1017/S0033291716001665.

14. Akca SO, Yuncu 0, Aydin Z. Mental status and suicide probability of young people: a cross-sectional study. Rev Assoc Med Bras. 2018;64(1):32-40. doi: https://doi.org/10.1590/1806-9282.64.01.32.

15. Li Y, Shi J. Bullying and suicide in high school students: findings from the 2015 California youth risk behavior survey. J Human Behav Soc Environ. 2018;13(4):44-56. doi: https://doi.org/10.1080/10911359.2018.1456389.
16. Martins-Monteverde CMS, Padovan T, Juruena MF. Transtornos relacionados a traumas e a estressores. Medicina (Ribeirao Preto). 2017;50(Supl.1):37-50. doi: https://doi.org/10.11606/issn.2176-7262.v50isup11.p37-50.

17. Gonçalves A, Freitas P, Sequeira C. Comportamentos suicidários em estudantes do ensino superior: factores de risco e de protecção. Millenium. 2016 [citado 2018 Jan 11];40:149-59. Disponível em: http://revistas.rcaap.pt/millenium/ article/view/8225/5840.

18. Luca SM, Franklin C, Yuegi Y, Johnson S, Brownson C. The relationship between suicide ideation, behavioral health, and college academic performance. Community Ment Health J. 2016;52(5):534-40. doi: https://doi.org/10.1007/ s10597-016-9987-4.

19. Blasco MJ, Castellví P, Almenara J, Lagares C, Roca M, Sesé A, et al. Predictive models for suicidal thoughts and behaviors among Spanish University students: rationale and methods of the UNIVERSAL (University \& mental health) project. BMC Psychiatry. 2016;16:122. doi: https://doi.org/10.1186/s12888-0160820-y.

20. González-Sepúlveda PC, Medina-Pérez OA, Ortiz-Valencia JC. Riesgo suicida y factores asociados en estudiantes de Psicología en una universidad pública de Colombia. Rev Haban Cienc Méd. 2016 [citado 2018 Mayo 29];15(1):136-46. Disponible en: http://scielo.sld.cu/scielo.php?script=sci_arttext\&pid=\$1729519X2016000100015\&lng=es.

\section{- Autor correspondente:}

Fernando José Guedes da Silva Júnior E-mail: fernandoguedes@ufpi.edu.br
Recebido: 19.06 .2018

Aprovado: 09.05.2019 